\title{
Can the new epidemiology of schizophrenia help elucidate its causation?
}

\author{
R. T. Abed ${ }^{1, *}$ and M. J. Abbas ${ }^{2}$ \\ ${ }^{1}$ Mental Health Tribunals, Sheffield, UK \\ ${ }^{2}$ Leicestershire Partnership NHS Trust, Leicester, UK
}

\begin{abstract}
The supposed universality of the incidence and prevalence of schizophrenia has been seriously challenged. It is now widely accepted that the life-time prevalence and incidence of this disorder vary considerably in time and place. As a result, there has been renewed interest in environmental causation of schizophrenia. There are few extant formulations that have successfully integrated the available new evidence into a coherent theory for its causation. The outgroup intolerance hypothesis is an attempt to integrate this evidence. It proposes that schizophrenia is the result of a mismatch between the social brain as shaped by evolution and the new social conditions of the post-neolithic. The hypothesis can provide an explanation for (i) the higher risk to migrants, (ii) the ethnic density phenomenon, (iii) the increased risk to individuals who have grown up in cities and (iv) the putative low risk in hunter-gatherer societies. Evidence is presented from a range of disciplines and sources including epidemiology, psychopathology, social psychology and clinical trials in support of this hypothesis. A range of testable predictions follow from the hypothesis.
\end{abstract}

Received 26 June 2013; Revised 1 October 2013; Accepted 15 January 2014

Key words: Environmental factors, epidemiology, evolutionary theory, outgroup, schizophrenia.

Any theory that attempts to integrate the new epidemiological data on schizophrenia must attempt to identify the precise nature of the environmental stressor(s), be consistent with the new epidemiological data, be consistent with findings on schizophrenia in spheres other than epidemiology and should make predictions about the disorder that are testable and are currently unknown.

There is now strong evidence that the prevalence and incidence of schizophrenia vary according to gender, place, urbancity, migration status and ethnic density (Krabbendam \& van Os, 2005; McGrath et al. 2008; Das-Munshi et al. 2012). In summary, the risk is higher in males, in first- and second-generation immigrants, in urban settings and in minority populations living in an area with a low owngroup ethnic density.

This new evidence has led to a renewed interest in the role of the environmental and, especially, social factors in the causation of schizophrenia. A number of theories have been proposed. For example, the selective migration theory (Odegaard, 1932) was an early attempt to explain the higher rate among immigrants. The role of nutritional factors (McGrath, 1999), infection (Squires, 1997), environmental pollution (Pedersen \& Mortensen, 2006) and stress because of overcrowding (Cougnard et al. 2007). However, none of these theories

* Address for correspondence: R. T. Abed, FRCPsych, 17 Kenwood Bank, Sheffield S7 1NU, UK.

(Email: abedrt@btinternet.com) can satisfactorily account for the new epidemiological findings on this disorder.

Another example of an environmental theory is the social defeat hypothesis (Selten \& Cantor-Graae, 2007), which suggested that it is the cumulative impact of a number of disadvantageous situations leading to a defeat situation that leads to schizophrenia. However, this hypothesis lacks specificity and can apply to all mental disorders both psychotic and non-psychotic. Therefore, while undoubtedly an important stress factor for mental disorder in general it does not provide a satisfactory explanation for schizophrenia.

The variability in prevalence and incidence was also problematic for some evolutionary formulations of schizophrenia that suggested either a hidden evolutionary advantage (for example Kuttner et al. 1967; Allen \& Sarich, 1988; Sullivan \& Allen, 1999) or that the condition arises as a by-product of particular human brain systems such as language (Crow, 1997) or the social brain (Burns, 2004). Burns, in an attempt to accommodate this new evidence of variability, suggested a gene-environment approach. The genes for psychosis are seen as 'normal genes' that play a role in development and that 'a spectrum of genetic vulnerability exists in the population, which in the context of a toxic social environment is expressed as a continuum of psychosis' (Burns, 2009). The 'toxic social environment' has been linked to being male, immigrant, living in urban city or in an area of low ethnic density but both the nature of the pathogenic environmental factor and the genetic vulnerability remained poorly defined. 
We have proposed elsewhere (Abed \& Abbas, 2011) that outgroup intolerance helped both define the offending environmental stressor as well as the preexisting vulnerability. In this brief paper we provide a further elaboration of the hypothesis by specifying the nature of the putative vulnerability in individuals who develop schizophrenia. We also discuss new research not available to us when the original hypothesis was published. This includes new findings on the role of ethnic density in influencing the risk of schizophrenia and also further evidence regarding 'visible ethnic minority status' as a risk factor. The new evidence confirms and strengthens the findings of previous research and is highly supportive of the outgroup intolerance hypothesis $(\mathrm{OIH})$. We also briefly consider the potential implications for the recent findings of the role of oxytocin in modulating cooperation and competition and in creating intergroup bias with primarily ingroup favouratism and solidarity. And our final aim is to summarise $\mathrm{OIH}$ in a short accessible paper that would reach a wider audience who may be put off by a lengthy theoretical article. We hope by doing this we can generate interest in the evolutionary approach and hopefully generate interest in testing some of our hypotheses and predictions.

The OIH is based on the assumption that humans evolved for most of their history in hunter-gatherer societies, characterised by close kinship and a clear demarcation between ingroup and outgroup membership. It follows, therefore, that the human brain evolved in such a way as to expect input from a social environment that has a high density of kin and ingroup members during critical stages of development. Kin and ingroup members are likely to provide care, comfort and support as opposed to outgroup individuals, who would be more likely to be hostile and/or dangerous. Departure from this ancestral social configuration into one where there is an abundance of outgroup members and/or paucity of ingroup members leads to distress and this, in turn, can lead to aberrant development of the social brain in vulnerable individuals. Hence, the nature of the pathogenic environmental stressor is either a paucity of kin or ingroup members or an abundance of outgroup members in the social environment during critical stages of development or some combination of both. Also, the hypothesised nature of the vulnerability is either an over-sensitivity to the existence of strangers (outgroup individuals) in the social environment or an impairment in the individual's ability to re-categorise or re-designate outgroup individuals as ingroup members or some combination of both. However, it is hypothesised that the vulnerability to schizophrenia although ancient, remained completely or largely hidden in the original human environment and did not become manifest until the post-neolithic when living in the vicinity of strangers became increasingly common (Abed \& Abbas, 2011). According to this model, the 'spectrum of genetic vulnerability' is hypothesised to be in the genes that regulate the neurobiological system that process information related to ingroup-outgroup membership and interactions.

The evidence for this hypothesis is as follows

1. First- and second-generation immigrants are at a higher risk of psychosis. The finding that migrants are at greater risk has been confirmed by more than one meta-analysis; moreover, the risk was found to rise further if the migrants are racially distinct from the host population (Cantor-Graae \& Selten, 2005; Bourque et al. 2011). The increased risk persists in second-generation migrants where the effect of racial visibility becomes even more pronounced. However, the risk of psychosis is cancelled out if the migration involves a move from a situation of social exclusion to social inclusion (van Os, 2012). It is evident that these findings are consistent with and supportive of $\mathrm{OIH}$.

2. Urban living is a risk factor (Krabbendam \& van Os, 2005). Exposure to outgroup members is expected to be higher in cities compared to rural areas. Also, the dose-response relationship of the time spent in an urban setting during early upbringing (van Os, 2012) is supportive of OIH as the greater the exposure to the outgroup-dense environment during critical stages of development the greater the expected risk.

3. The evidence from studies of the risk in ethnic minorities suggest that there is a greater increase in risk in more visible minorities (van Os, 2012) and the risk decreases with an increase in same-group ethnic density within a given locality (Das-Munshi et al. 2012).

4. The variable rate of incidence and prevalence of schizophrenia in different countries. There is some evidence that appears to point towards lower rates of schizophrenia in developing countries with more traditional societies and higher rates in westernised, industrialised and highly urbanised ones (Torrey et al. 1974; Egeland \& Hostetter, 1983; Torrey, 1987; McGrath et al. 2008).

5. There is evidence that the prognosis of schizophrenia is improved in developing countries despite poor or non-existent mental health services (World Health Organization, 1973; Sartorius et al. 1986; Jablensky et al. 1992). This finding could be supportive of the $\mathrm{OIH}$ if the more benign course can be shown to be related to a less-pathogenic social environment by reason of its greater resemblance to the human environment of evolutionary adaptedness (EEA). 
6. Higher prevalence in males (McGrath et al. 2008). In the human ancestral environment the danger from outgroup individuals emanated primarily if not exclusively from males and their main victims were other males. It is of interest that studies have found that men in contemporary societies display more xenophobic and ethnocentric attitudes than do women, which has been consistent across cultures, samples and time (McDonald et al. 2012). Men also dehumanise and use danger-relevant stereotypes about outgroup members (McDonald et al. 2012) all of which are supportive of OIH. This biological propensity may place males under greater stress in environments where there is a high density of outgroup members. However, we accept that the excess of risk to males is lower than would be predicted by $\mathrm{OIH}$.

7. The age of onset of schizophrenia is in late adolescence and early adulthood (Hafner et al. 1994). It is during this period that human development involves a shift from kin to non-kin interactions, which is considered to be an important milestone in human development (Alcorta, 2009).

8. The therapeutic effect of oxytocin in schizophrenia. A recent review (MacDonald \& Feifel, 2012) concluded that oxytocin acts as a therapeutic agent in schizophrenia, that it has an antipsychotic action and also, that it is implicated in the mechanism of action of clozapine. When this is linked with other evidence that suggests that oxytocin provides the biological basis for ingroup solidarity, trust, cooperation, favouratism and ethnocentrism and indirectly causes outgroup disfavour and hostility (De Dreu et al. 2010, 2011), it becomes evident that these findings are potentially supportive of the $\mathrm{OIH}$. This raises the question as to whether the specific action of oxytocin in increasing ingroup affiliative behaviour may have the potential of permanently correcting the putative neurobiological deficit (according to OIH) in the early stages of schizophrenia where the threshold for designating others as members of the ingroup is set too high and thus causing an excess of outgroup designations. If this is the case, oxytocin could have the potential of arresting the progress of the illness if used early enough. Also, oxytocin may have a preventative role in ultra high-risk individuals. We believe that these are plausible hypotheses that can be answered through well-designed research trials.

9. Psychopathology: we would contend that the core psychopathology of schizophrenia involves hypervigilance and overattribution of malicious intentions to others. This suggests that in schizophrenia mechanisms involved in the evaluation of social threats are overactive (Brüne, 2008). A large proportion of positive symptoms, such as delusions and hallucinations, relate to the theme of threat, which in the ancestral environment would have been likely to have had its origin external to the group. Persecutory delusions in males were found in one particular study to relate mostly to gangs of male strangers (Zolotova \& Brüne, 2006), which is consistent with OIH. Also, it is of particular interest that the vast majority of auditory hallucinations are of male voices (Nayani \& David, 1996). The evidence also suggest that intergroup bias (favouring ingroup members and hostility to outgroups) is primarily directed at men (McDonald et al. 2012) and hence males are both the primary perpetrators as well as victims. The negative symptoms may represent a withdrawal of the person from social contact. We accept, however, that some of the psychopathology, for example, grandiosity, bizarre delusions and disorganised thinking does not easily fit into this model.

10. There is strong evidence that childhood abuse is a risk factor for schizophrenia (Varese et al. 2012). The perpetrators can be both biological kin as well as non-kin. However, Daly \& Wilson (1985) found that the risk of abuse rose 40 -fold when children lived with one stepparent and one biological parent compared to children living with two biological parents. Indeed, stepparenting is a significant risk factor leading to child abuse and homicide (Buss, 1999). The association of being parented by non-kin with a higher risk of being abused that in turn increases the risk of schizophrenia would be consistent with the $\mathrm{OIH}$. This raises the question as to whether being parented by non-kin is, in itself, a risk factor for schizophrenia. Clearly, this question can only be answered by empirical research.

While the $\mathrm{OIH}$ is primarily a hypothesis of environmental causation of schizophrenia it is unambiguously biological. It assumes a gene-environment interaction that can have profound biological consequences for brain and psychological development. We propose here a broad concept of biology (consistent with Darwinian principles) that goes beyond the narrow reductionist view that limits it to events at the molecular or cellular level. Therefore, social and environmental factors that interact in specific ways with the human organism to shape the individual phenotype can only be properly understood when viewed as part of a biological process. $\mathrm{OIH}$ assumes that the pathogenic social environment of modern advanced societies interacts with neurobiological systems leading to aberrant development of the social brain resulting in schizophrenia. Hence, the $\mathrm{OIH}$ is potentially compatible with the structural brain changes found in schizophrenia and also with future discoveries 
of microscopic brain pathology. The $\mathrm{OIH}$ proposes a number of testable predictions about the prevalence and prognosis of schizophrenia in relation to the degree to which a given society departs from the conditions of the EEA or ethnic density or cultural integration. It also proposes that patients with schizophrenia and possibly first-degree relatives will show greater evidence of sensitivity to outgroup individuals than the average person (Abed \& Abbas, 2011).

The OIH has considerable explanatory power and makes a number of predictions that are testable and refutable. However, we recognise that further work on the definition of the precise nature of outgroup intolerance and its measurement is needed. A research design that accommodates a gene-environment model would significantly improve the probability of supporting or refuting this hypothesis.

\section{References}

Abed RT, Abbas MJ (2011). A reformulation of the social brain theory for schizophrenia: the case for out-group intolerance. Perspectives in Biology and Medicine 54, 132-151.

Alcorta CS (2009). Religious behaviour and the adolescent brain. In The Biology of Religious Behavior: The Evolutionary Origins of Faith and Religion (ed. J. R. Feierman), pp. 106-122. Praeger: Santa Barbara.

Allen JS, Sarich VM (1988). Schizophrenia in an evolutionary perspective. Perspectives in Biology and Medicine 32, 132-153.

Bourque F, van der Ven E, Malla A (2011). A meta-analysis of the risk for psychotic disorders among first- and secondgeneration immigrants. Psychological Medicine 41, 897-910.

Brüne M (2008). Textbook of Evolutionary Psychiatry: The Origins of Psychopathology. Oxford University Press: Oxford.

Burns JK (2004). An evolutionary theory of schizophrenia: cortical connectivity, metarepresentation, and the social brain. Journal of Behavioral and Brain Science 27, 831-855, discussion 855-885.

Burns JK (2009). Reconciling 'the new epidemiology' with an evolutionary genetic basis for schizophrenia. Medical Hypotheses 72, 353-358.

Buss DM (1999). Evolutionary Psychology: The New Science of the Mind. Allyn and Bacon: Boston.

Cantor-Graae E, Selten JP (2005). Schizophrenia and migration: a meta-analysis and review. American Journal of Psychiatry 162, 12-24.

Cougnard A, Marcelis M, Myin-Germeys I, De Graaf R, Vollebergh W, Krabbendam L, Lieb R, Wittchen HU, Henquet C, Spauwen J, Van Os J (2007). Does normal developmental expression of psychosis combine with environmental risk to cause persistence of psychosis? A psychosis proneness-persistence model. Psychological Medicine 37, 513-527.

Crow TJ (1997). Is schizophrenia the price that Homo sapiens pays for language? Schizophrenia Research 28, 127-141.

Daly M, Wilson M (1985). Child abuse and other risks of not living with both parents. Ethology and Sociobiology 6, 197-210.
Das-Munshi J, Becares L, Boydell JE, Dewey ME, Morgan C, Stansfeld SA, Prince MJ (2012). Ethnic density as a buffer for psychotic experiences: findings from a national survey (EMPIRIC). British Journal of Psychiatry 201, 282-290.

De Dreu CK, Greer LL, Handgraaf MJ, Shalvi S, Van Kleef GA, Baas M, Ten Velden FS, Van Dijk E, Feit SW (2010). The neuropeptide oxytocin regulates parochial altruism in intergroup conflict among humans. Science 328, 1408-1411.

De Dreu CK, Greer LL, Van Kleef GA, Shalvi S, Handgraaf MJ (2011). Oxytocin promotes human ethnocentrism. Proceedings of the National Academy of Sciences of the United States of America 108, 1262-1266.

Egeland JA, Hostetter AM (1983). Amish study, I: affective disorders among the Amish, 1976-1980. American Journal of Psychiatry 140, 56-61.

Hafner H, Maurer K, Loffler W, Fatkenheuer B, an der Heiden W, Riecher-Rossler A, Behrens S, Gattaz WF (1994). The epidemiology of early schizophrenia. Influence of age and gender on onset and early course. The British Journal of Psychiatry Supplement 23, 29-38.

Jablensky A, Sartorius N, Ernberg G, Anker M, Korten A, Cooper JE, Day R, Bertelsen A (1992). Schizophrenia: manifestations, incidence and course in different cultures. A World Health Organization ten-country study. Psychological Medicine Monograph Supplement 20, 1-97.

Krabbendam L, van Os J (2005). Schizophrenia and urbanicity: a major environmental influence-conditional on genetic risk. Schizophrenia Bulletin 31, 795-799.

Kuttner RE, Lorincz AB, Swan DA (1967). The schizophrenia gene and social evolution. Psychological Reports 20, 407-412.

MacDonald K, Feifel D (2012). Oxytocin in schizophrenia: a review of evidence for its therapeutic effects. Acta Neuropsychiatrica 24, 130-146.

McDonald MM, Navarrete CD, Van Vugt M (2012). Evolution and the psychology of intergroup conflict: the male warrior hypothesis. Philosophical Transactions of the Royal Society of London. Series B, Biological Sciences 367, 670-679.

McGrath J (1999). Hypothesis: is low prenatal vitamin D a risk-modifying factor for schizophrenia? Schizophrenia Research 40, 173-177.

McGrath J, Saha S, Chant D, Welham J (2008). Schizophrenia: a concise overview of incidence, prevalence, and mortality. Epidemiological Review 30, 67-76.

Nayani TH, David AS (1996). The auditory hallucination: a phenomenological survey. Psychological Medicine 26, 177-189.

Odegaard O (1932). Emigration and insanity: a study of mental disease among the Norwegian-born population of Minnesota. Acta Psychiatrica Scandinavica 4, 1-206.

Pedersen CB, Mortensen PB (2006). Urbanization and traffic related exposures as risk factors for schizophrenia. $B M C$ Psychiatry 6, 2.

Sartorius N, Jablensky A, Korten A, Ernberg G, Anker M, Cooper JE, Day R (1986). Early manifestations and firstcontact incidence of schizophrenia in different cultures. A preliminary report on the initial evaluation phase of the WHO Collaborative Study on determinants of outcome of severe mental disorders. Psychological Medicine 16, 909-928. 
Selten JP, Cantor-Graae E (2007). Hypothesis: social defeat is a risk factor for schizophrenia? British Journal of Psychiatry Supplement 51, s9-s12.

Squires RF (1997). How a poliovirus might cause schizophrenia: a commentary on Eagles' hypothesis. Neurochemical Research 22, 647-656.

Sullivan RJ, Allen JS (1999). Social deficits associated with schizophrenia defined in terms of interpersonal Machiavellianism. Acta Psychiatrica Scandinavica 99, 148-154.

Torrey EF (1987). Prevalence studies in schizophrenia. British Journal of Psychiatry 150, 598-608.

Torrey EF, Torrey BB, Burton-Bradley BG (1974). The epidemiology of schizophrenia in Papua New Guinea. American Journal of Psychiatry 131, 567-573. van Os J (2012). Psychotic experiences: disadvantaged and different from the norm. British Journal of Psychiatry 201 258-259.

Varese F, Smeets F, Drukker M, Lieverse R, Lataster T, Viechtbauer W, Read J, van Os J, Bentall RP (2012). Childhood adversities increase the risk of psychosis: a meta-analysis of patient-control, prospective- and cross-sectional cohort studies. Schizophrenia Bulletin 38, 661-671.

World Health Organization (1973). Report of the International Pilot Study of Schizophrenia. WHO: Geneva.

Zolotova J, Brüne M (2006). Persecutory delusions: reminiscence of ancestral hostile threats? Evolution and Human Behavior 27, 185-192. 